\title{
Ambulatory assessment of shoulder abduction strength curve using a single wearable inertial sensor
}

\author{
Pietro Picerno, PhD ( $^{*}$ Valerio Viero, MSc; ${ }^{2}$ Marco Donati, PhD; ${ }^{3}$ Tamara Triossi, MSc; ${ }^{2}$ Virginia Tancredi, \\ PhD; ${ }^{2}$ Giovanni Melchiorri, PhD $^{2,4}$ \\ ${ }^{1}$ Faculty of Psychology, School of Sport and Exercise Sciences, eCampus University, Novedrate, Italy; and Faculty of \\ Medicine and Surgery, School of Sport and Exercise Sciences, University of Rome Tor Vergata, Rome, Italy; \\ ${ }^{2}$ Department of Medicine Systems, Faculty of Medicine and Surgery, University of Rome Tor Vergata, Rome, Italy; \\ ${ }^{3}$ Research and Development, Sensorize, Rome, Italy; ${ }^{4}$ Don Carlo Gnocchi Foundation, Milano, Italy
}

\begin{abstract}
The aim of the present article was to assess the reliability of strength curves as determined from tridimensional linear accelerations and angular velocities measured by a single inertial measurement unit (IMU) fixed on the upper arm during a shoulder abduction movement performed holding a $1 \mathrm{~kg}$ dumbbell in the hand. Within-subject repeatability of the task was assessed on 45 subjects performing four trials consisting of one maximal shoulder abduction-adduction movement. Intraclass correlation coefficient (ICC) was computed on the average movement angular velocity (VEL) and range of movement (ROM) across the four trials. Within-subject repeatability of torque curves was assessed in terms of waveform similarities by computing the coefficient of multiple determination (CMD). Accuracy of the estimated ROM was assessed using an isokinetic dynamometer. High ICC values of ROM (0.955) and VEL (0.970) indicated a high within-subject repeatability of the task. A high waveform similarity of torque curves was also found between trials $(\mathrm{CMD}=0.867)$. Accuracy with respect to isokinetic dynamometer in estimating ROM was always $<1$ degree $(p=0.37)$. This study showed the effectiveness of using a single wearable IMU for the assessment of strength curve during isoinertial movements in a way that complies with the needs of clinicians in an ambulatory setting.
\end{abstract}

Key words: accelerometers, biomechanics, gyroscopes, inertial sensors, injury recovery, isoinertial, joints, rehabilitation, shoulder abduction, strength curves.

\section{INTRODUCTION}

Due to muscle fiber (sarcomere length) and joint (muscle force moment arm) mechanics, the capacity of muscles to produce force varies as a function of joint angle. The graphical representation of this relation has been defined as the strength curve [1]. Muscle strength (dependent variable) is generally represented by external forces/torques measured using dynamometers while a joint lever is attempting to rotate against an external resistance.

When one is dealing with an injured joint, knowledge of force production patterns throughout the range of motion (ROM) becomes essential for assessing damages, monitoring functional recovery, and addressing therapeutic interventions avoiding overloads of the muscle-tendon structure during the rehabilitation phase. For this reason,

Abbreviations: $3 \mathrm{D}=$ tridimensional, $\mathrm{BMI}=$ body mass index, $\mathrm{CI}=$ confidence interval, $\mathrm{CMD}=$ coefficient of multiple determination, ICC = intraclass correlation coefficient, IMU = inertial measurement unit, $\mathrm{ROM}=$ range of motion, $\mathrm{SE}=$ systematic error, VEL = angular velocity.

*Address all correspondence to Pietro Picerno, PhD; eCampus University-Main Campus, via Isimbardi 10, 22060 Novedrate (CO), Italy; +39-0317942500-7942505; fax +39-031-792631. Email: pietro.picerno@gmail.com http://dx.doi.org/10.1682/JRRD.2014.06.0146 
strength curves have been mostly adopted in the field of rehabilitation and characterized using either load cells (by measuring isometric force at any given angle) or isokinetic dynamometers [2-4] as follows. Some studies have proved the effectiveness of using angle-specific force/torque curves in identifying and monitoring rehabilitation in pathologies of the patella [5]. In other studies, this approach has been used for analyzing anglespecific strength impairments of elbow flexors [6] or rotator cuff muscles [7-8]. Furthermore, strength curves have been adopted for characterizing muscle damage induced either by spinal cord injuries [9] or eccentric contraction in elbow flexors [10] and knee extensors [11]. Finally, strength curves have been used for assessing hamstring/quadriceps strength ratio [12] or for assessing general decrements in muscle function [13].

Beyond the effectiveness of strength curves as a clinical tool, from a practical point of view, their applicability is jeopardized by the use of isometric and isokinetic dynamometers, which are expensive and characterized by a cumbersome setup that is far from the needs of clinicians in an ambulatory setting. Furthermore, from a functional point of view, the muscle activation they produce is different from that characterizing typical human movements, which are performed neither with fixed joint angles nor at constant joint angular velocities [14].

Recent technological advances in the field of motion measurement techniques have opened up new perspectives in human movement analysis: instantaneous tridimensional (3D) linear accelerations and angular velocities of moving objects can be now measured directly on board using small inertial sensors such as accelerometers and gyroscopes, respectively [15]. The combination of a 3D accelerometer and gyroscope is usually referred to as an inertial measurement unit (IMU); linear accelerations and angular velocities are measured, respectively, along and about the geometrical axes of the IMU case, generally referred to as the local reference system. IMUs, typically used for estimating body segment orientation [16], have been specifically employed for the assessment of ROM during joint mobility tests (IMU fixed on the body segment) [17-19] and muscle strength during isoinertial strength tests (IMU fixed on the external resistance) $[17,20]$ by estimating the body segment's orientation and force impressed to the external resistance, respectively. This implies that ROM and strength of an injured joint while rotating against a constant external resistance (e.g., elbow flexion or shoulder abduction holding a dumbbell) can be potentially assessed using an "all-in-one" solution consisting of a single IMU, which would fully comply with the requirement of an in-field, ambulatory assessment. In particular, during an isoinertial resisted joint mobility test, an IMU fixed on the rotating body segment could be used to simultaneously estimate both the angular displacement and the angular acceleration of the segment: the first can be considered representative of joint ROM, while the second can be considered the result of a joint torque produced by the action of muscle forces.

The aim of the present study was to assess the reliability of using a single IMU for determining strength curves during isoinertial exercises. A shoulder abduction movement performed against an external resistance was chosen as the paradigm for introducing this novel methodological approach.

\section{METHODS}

\section{Experimental Protocol}

Forty-five subjects (25 males, age $27 \pm 8$ yr, body mass index [BMI] $23 \pm 3 \mathrm{~kg} / \mathrm{m}^{2}$; and 20 females, age $22 \pm 3 \mathrm{yr}$, BMI $21 \pm 2 \mathrm{~kg} / \mathrm{m}^{2}$ ) without any previous or current shoulder impairment and not involved in competitive sports at a professional level participated in the study. Before data collection, subjects were instructed on the task to perform. The task consisted of one consecutive shoulder abduction-adduction movement while holding a $1 \mathrm{~kg}$ dumbbell in the hand. The task was performed in a standardized seated position and started with the arm along the trunk. Subjects were asked to (1) perform the ascending part of the movement (abduction) as fast as possible, reaching the maximum joint angular excursion before inverting the rotation, and (2) keep the elbow fully extended and the wrist in a neutral position during the whole arc of movement. Finally, care was taken in instructing subjects to perform the movement purely on their frontal plane and without any compensatory movements such as lateral trunk bending that might alter the measure of shoulder abduction ROM.

\section{Strength Curve Determination}

The strength curve relative to the shoulder abduction movement was assessed using a wireless IMU (FreeSense, SENSORIZE; Rome, Italy) fixed with an arbitrary orientation on an arbitrary point of the upper 
arm using an elastic belt. Care was taken in fixing the IMU on the body segment in order to minimize, as much as possible, the relative motion of the IMU with respect to the segment (i.e., skin artifacts) [21-22]. Inertial sensors signals were sampled at $200 \mathrm{~Hz}$ and sent via Bluetooth to a laptop computer.

During a shoulder abduction/adduction movement, because sensors are $3 \mathrm{D}$, the direction of the vector of the measured 3D angular velocity $(\dot{\theta})$ coincides with the joint's single axis of rotation [23], independently from how the IMU case has been oriented when fixed on the body segment. The instantaneous angular displacement of the IMU $(\theta)$ about the axis of rotation $(O)$ was estimated through numerical integration of $\dot{\theta}$. To cope with time-increasing errors due to the numerical integration process [24], the sensor fusion algorithm proposed by Mahony et al. was used [25].

Only the ascending part of $\theta$ and the corresponding of $\dot{\theta}$, relative to the abduction movement, were considered for further analysis (Figure 1). The upper arm-forearm- hand-dumbbell was assumed to be a single rigid system $(S)$, and $\theta$ was considered representative of the shoulder abduction ROM. The net abducting muscular torque at the shoulder $(T)$ was estimated by Equation 1:

$$
T=\ddot{\theta} \cdot I_{S}^{O}
$$

where $\ddot{\theta}$, in radians per second squared, is the angular acceleration of $S$ estimated by numerical differentiation of $\dot{\theta}$ [26] and $I_{S}^{O}$ is the moment of inertia of $S$ about the axis of rotation $O$. Note that the so-computed $T$ is the sum of the moments of all the external forces that rotate $S$ about $O$, and hence, it also includes the rotational component due to gravity. $I_{S}^{O}$ was computed using the parallel axis theorem (Equation 2):

$$
I_{S}^{O}=\sum I_{n}^{C M}+m_{n} \cdot d_{n}^{2}
$$

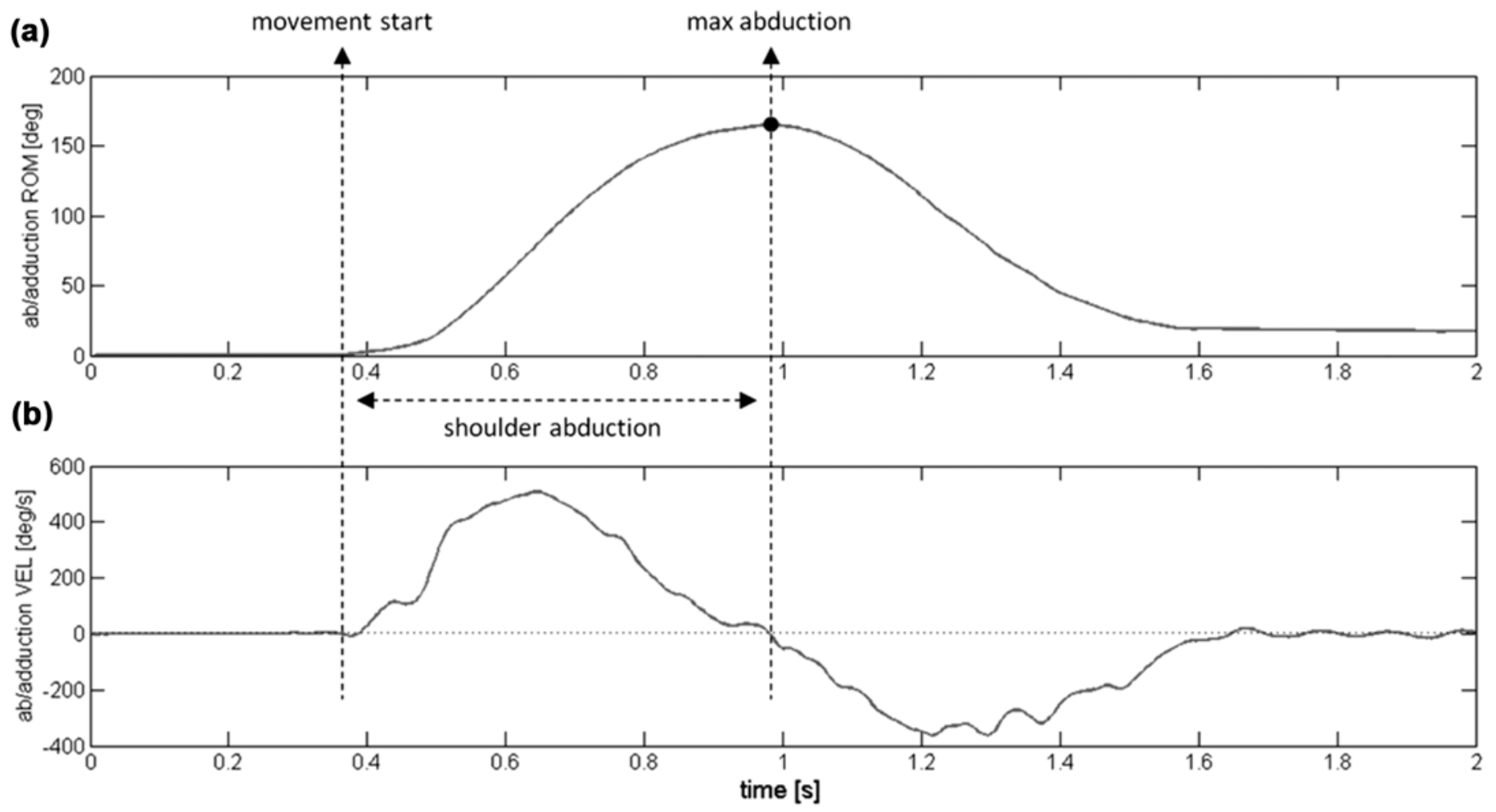

Figure 1.

Sample plots showing instantaneous angular displacement during consecutive shoulder abduction/adduction movement estimated by (a) inertial measurement unit and (b) relevant angular velocity (VEL). max = maximum, ROM = range of motion. 
as the sum of the moments of inertia of each of the $n$ segments of $S$ (upper arm, forearm, hand, and dumbbell) with respect to $O$. For each of the $n$ segments of $S$, the moment of inertia with respect to the segment's center of mass $\left(I^{C M}\right)$ is determined with respect to $O$ by adding to $I^{C M}$ the segment's mass $(m)$ multiplied by the squared distance from the segment's $C M$ to $O\left(d^{2}\right)$. The segment inertia parameters (mass, moment of inertia, and length) needed to solve the parallel axis theorem were estimated from the subjects' anthropometric data by using equations provided by de Leva [27], whereas the dumbbell's inertia parameters were computed from the geometry and mass of the dumbbell.

A strength curve relative to the shoulder abduction movement was, finally, obtained by plotting the torque $T$ as a function of the shoulder abduction angle $\theta$ (Figure 2). Note that the resulting strength curve is the result of an isoinertial muscle contraction [14], and hence, it represents the capacity of muscles to lift a constant external resistance (1 kg in this case) against gravity at the maximum voluntary angular acceleration. Different loads and angular accelerations (voluntary effort performed by the subject) yield different strength curve patterns and magnitudes.
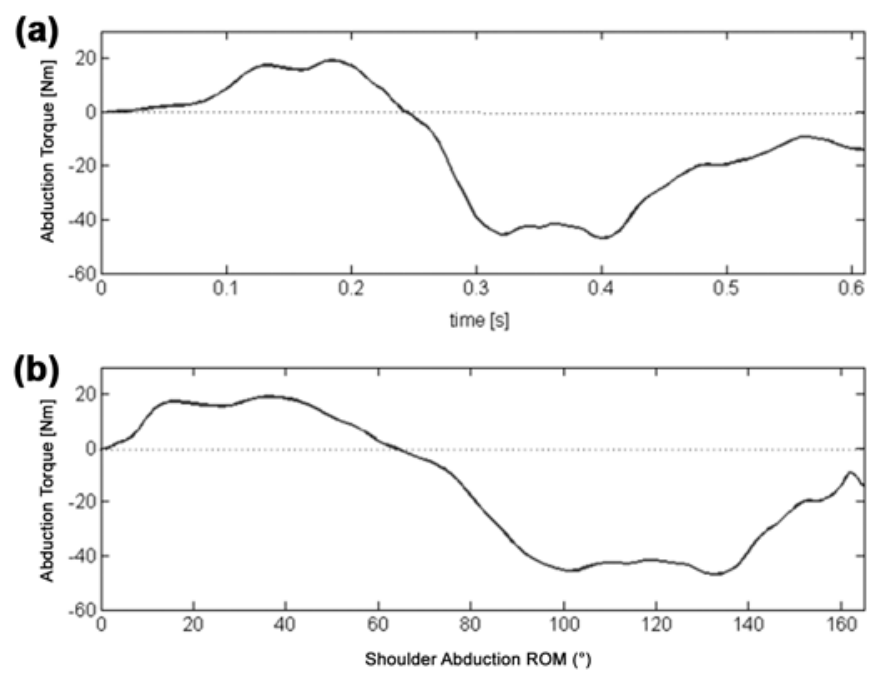

Figure 2.

Sample plots showing net shoulder abduction torque (from movement start to maximum abduction) as function of (a) abduction time and (b) abduction range of motion (ROM). The latter is also referred to as "strength curve."

\section{Repeatability Assessment}

After a specific warm-up, data collection began with subjects performing four trials consisting of one consecutive shoulder abduction-adduction movement each with their dominant arm. One minute of rest was taken between trials. Within-subject repeatability of the performed task was assessed by means of intraclass correlation coefficient (ICC) (type: ICC $(2,1)$ ) [28] computed on the average movement angular velocity (VEL) and ROM across the four trials. Within-subject repeatability of torque-time curves estimated in correspondence of the four trials was assessed by means of the coefficient of multiple determination (CMD) and systematic error (SE), the latter expressed as percentage of the range of the average between-trials torque-time curve [29-31]. The CMD assesses waveform similarity between timenormalized series of data [32], whereas SE is a measure of the overall difference between time-normalized series of data [33]. A CMD value, expressed as $r^{2}$, of 0 indicates no similarity and 1 indicates perfect agreement. CMD values $>0.75$ suggests good to excellent agreement between measurements of kinematic time curves [32]. Upper and lower 95 percent confidence intervals (CIs) were also reported for CMD and SE data.

\section{Accuracy Assessment}

Accuracy of the estimated angular displacement was assessed using an isokinetic dynamometer (Kin-Com, Isokinetic International; Chattanooga, Tennessee). One subject performed three consecutive shoulder abductionadduction movements at five different angular velocities (30, $60,90,120$, and $240 \%$ s) with $90^{\circ}$ of imposed ROM. The latter was used as reference for the ROM estimated using the IMU, which was fixed with an arbitrary orientation at the end of the rotating arm of the isokinetic machine (46 cm from the center of rotation) (Figure 3). Theoretically, errors introduced by the numerical integration process of a time series of data increase with time and noise-to-signal ratio; for this reason, the influence of number of repetitions and angular velocities on the estimated ROM was assessed using an analysis of variance for repeated measurements (within = repetitions, between = angular velocities). Furthermore, a Pearson correlation analysis was used to assess any trend between the error in estimating the ROM and the increasing VEL used in the experiments. Finally, a one-sample Student $t$-test was used to compare the estimated ROM against the true 


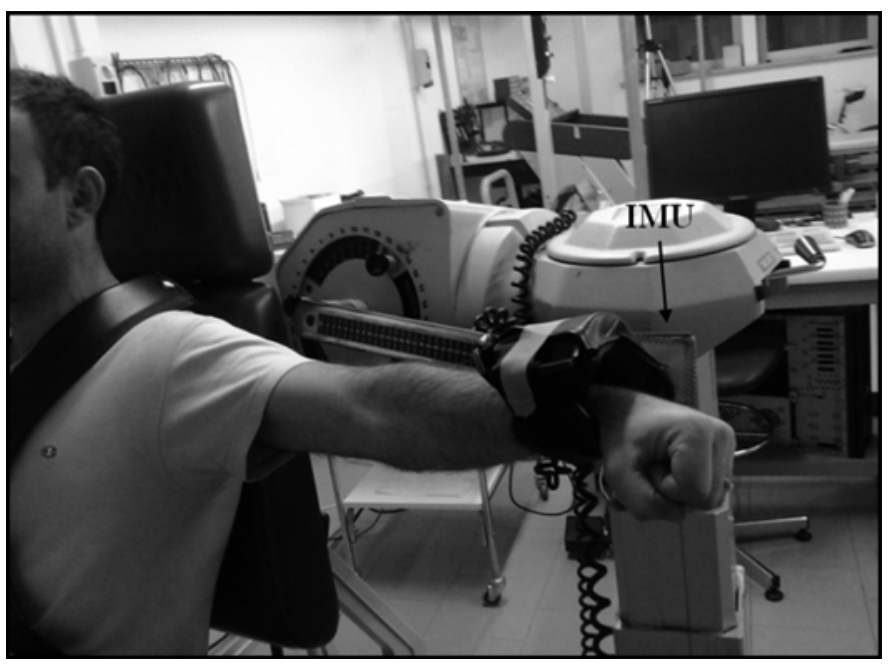

Figure 3.

Experimental setup used for accuracy assessment in estimating angular displacement using inertial measurement unit (IMU).

value $\left(90^{\circ}\right)$ for each single repetition of each of the five angular velocities. Significance for all statistical tests was set at $p<0.05$. Statistical analysis and signal processing were carried out using SPSS version 20 (IBM Corporation; Armonk, New York) and MATLAB R2011b (MathWorks; Natick, Massachusetts), respectively.

\section{RESULTS}

\section{Repeatability Assessment of Method}

Average ICC values relative to ROM and VEL computed between the four performed trials were 0.955 (95\% $\mathrm{CI}=0.930-0.979)$ and $0.97(95 \% \mathrm{CI}=0.952-0.982)$, respectively. Between-trial ROM and VEL standard deviations, expressed as percentage of the mean ROM and VEL collected over the four trials, were averaged over the 45 subjects and were equal to 3.3 and 4.8 percent, respectively.

CMD values, as computed between torque-time curves collected in correspondence of the four performed trials and considered over the 45 subjects, ranged from 0.846 to 0.888 (mean $=0.867$ ), whereas SE values ranged from 3.8 to 4.7 percent (mean $=4.2 \%$ ) of the range of the average between-trial torque-time curve.

\section{Accuracy Assessment of Method}

Mean error of the current method in estimating ROM was always $<1^{\circ}(p=0.37)$ in any of the $3 \times 5$ repetitions performed at the isokinetic dynamometer. Neither the number of repetitions $(p=0.21)$ nor the angular velocities $(p=0.62)$ were found to influence the estimated ROM. The latter did not correlate with the increasing angular velocities used in the experiments $(r=0.32)$. Figure 4 shows the accuracy of the current method in tracking instantaneous joint angular displacement with respect to that measured by the isokinetic dynamometer.

\section{DISCUSSION}

A strength curve is a powerful tool employed by clinicians for the assessment of muscle damage and functional recovery during a rehabilitation program [2,5]; targeted consequent therapeutic interventions may, indeed, rely on the knowledge of strength capability of muscles at any given angle of the joint's arc of movement. Strength curves have been also used by researchers to analyze muscle damage related to different types of muscle contractions or activities [9-11,13]. This is typically accomplished by means of isometric or isokinetic dynamometry. This study explored the feasibility and reliability of assessing strength curves during isoinertial movements using a single wearable device based on 3D linear accelerometer and gyroscope sensors. In particular,

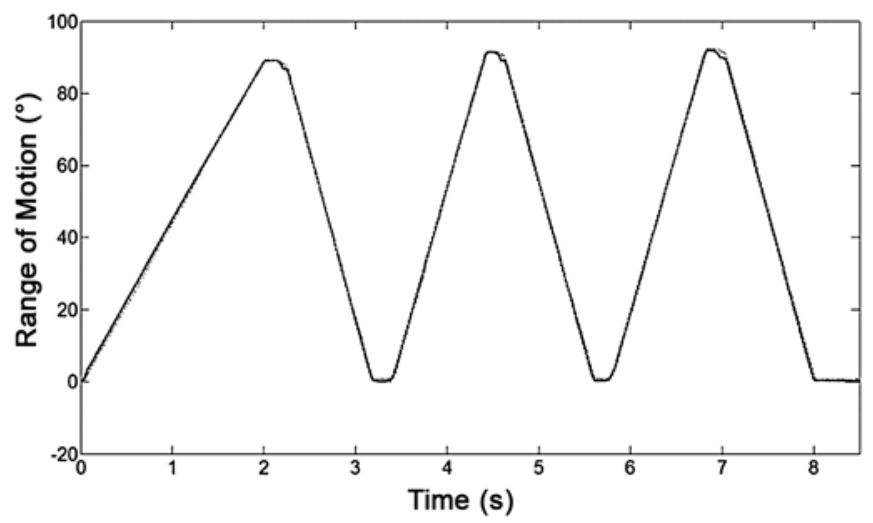

Figure 4.

Plot showing instantaneous angular displacement as measured by the isokinetic dynamometer (black line) and estimated by inertial measurement unit (gray line) during three consecutive shoulder abduction-adduction movements performed at $90 \%$ s. 
a shoulder abduction movement performed against a $1 \mathrm{~kg}$ dumbbell was chosen as the paradigm for introducing this novel methodological approach.

First, we wanted to ensure that the task, a shoulder abduction movement holding a $1 \mathrm{~kg}$ dumbbell in the hand, was repeatable if assessed over four different trials by 45 subjects. To do so, we analyzed the consistency of the ROM and movement's average VEL between the four shoulder abduction movements performed by each subject; the high reproducibility of the task was confirmed by high ICC values $(\mathrm{ROM}=0.955$, VEL $=0.970)$ and low between-trial standard deviation $(\mathrm{ROM}=3.3 \%$ and VEL $=$ $4.8 \%$ of the mean ROM and VEL collected over the four trials). Then, within-subject repeatability of exerted net abduction torque was assessed in terms of waveform similarity between torque-time curves as collected over the four trials; according to Kadaba et al. [32], a mean CMD value of 0.867 reflects good to excellent agreement between measurements of torque-time curves (Figure 5). An average SE of 4.2 percent revealed, as well, a low offset between the four torque-time curves collected for each subject.

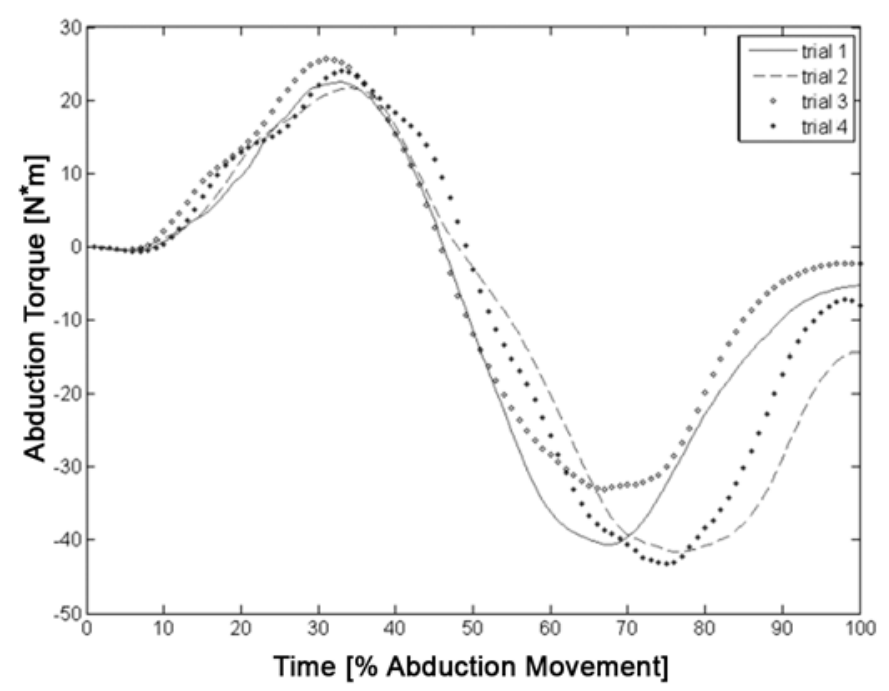

Figure 5.

Sample plot relative to 1 of 45 subjects in study showing intertrial repeatability of time-normalized torque curves (expressed as percentage of shoulder abduction movement) collected in correspondence of four performed trials. For this sample subject, coefficient of multiple determination $=0.845$ and rootmean-square error $=3.6 \mathrm{~N} \cdot \mathrm{m}$; the latter corresponds to $5.7 \%$ of average range of torque curves $(63.1 \mathrm{~N} \cdot \mathrm{m})$.
Finally, accuracy assessment was carried out. Because the main weakness of using miniature inertial sensors is a low frequency, unpredictable drift affecting data derived from numerical integration of sensor output [24], the instantaneous shoulder abduction/adduction angular displacement estimated through numerical integration of the measured VEL was compared to that measured by an isokinetic dynamometer at different VELs. The analysis revealed the high accuracy of the current method (error was found to always be $<^{\circ}$ ) and no statistical differences between the estimated and measured angular displacements. This successful result was not surprising because of the well-established efficacy of most of the sensor fusion algorithms proposed in the last decade.

With regard to other data estimated by numerical calculus, future works may lead on assessing accuracy of the angular acceleration derived through numerical differentiation of the measured VEL. The error introduced by such numerical process is a high frequency noise (i.e., spikes) in the derived signal that can be filtered out either by lowpass filtering the original signal before the numerical differentiation process or by performing the numerical differentiation using smoothing spline [26] or wavelet functions [34]. This source of error, although much more manageable than that related to numerical integration, needs to be further investigated because all the proposed solutions in the literature are mostly based on linear positional data collected by camera-based movement analysis systems [26] and little is known about managing numerical differentiation of inertial sensors signals.

If standardization of protocols for the assessment of strength curves is, generally, a fundamental requirement for the sake of repeatability and cross-comparison of results (past attempts to define normative strength curves for shoulder failed because of a lack of standardization, e.g., plane of motion, position of the shoulder, or stabilization of the body [35]), this becomes critical when tests are performed in isoinertial mode making use of a single IMU. With regard to joint mobility assessment, while use of a single IMU makes the measurement setup ambulatory and time- and cost-effective, special care must be taken in the execution of the movement in order to avoid substitute movements or compensations that may result in false or unwanted joint measurements (e.g., elbow flexion during shoulder abduction movement), respectively. This risk, in fact, could be avoided using multiple IMUs on adjacent body segments. With regard to the assessment of muscle strength, the prerequisite of any 
strength test is to make sure that the subject performs the test with maximal voluntary effort; this condition, easier to obtain in isokinetic and isometric modality (because the subject is not asked to control the movement because the latter is either guided or performed against a fixed resistance, respectively), is less controllable for isoinertial strength test. The only solution is to ask the subject to repeat the test several times and then check the repeatability of strength values. In the current study, this problem was addressed by evaluating waveform similarity of torque-time curve (strength assessment) and repeatability of ROM and VEL measurements (joint mobility assessment). Both analyses revealed high reproducibility of the task, both in movement kinematics and exerted effort.

Finally, beyond all the advantages of assessing strength curves in isoinertial mode using a single wireless IMU (consistent muscle contraction, cost-effective instrumentation, ambulatory evaluation, etc.), the clinical effectiveness of assessing strength curves in isoinertial mode still needs to be explored. Moreover, there are also some methodological issues that would require further analysis: (1) a mathematical-related issue may concern the characterization of the error related to the numerical differentiation of the measured angular velocity (which is directly related to the accuracy of the estimated net joint moment); (2) a task-related issue may concern the identification of the possible error associated with the estimate of planar joint kinematics due to errors in the execution of the movement (substitute movements or compensations may result in false or unwanted joint measurements) when only a single sensor is used; and (3) finally, since the isoinertial strength curve is load specific, a protocol-related issue may concern the choice of the amount of external load that has to be used for the assessment according to the type of disease and patient.

\section{CONCLUSIONS}

This study showed that a joint's strength curve can be determined not only using isokinetic or isometric dynamometry but also during functional movements using a single IMU. This may open up new perspectives for clinicians because a strength curve could then be easily obtained in ambulatory settings and, hence, used as a routinary diagnostic tool for assessing the functional recovery of a joint. In this regard, clinical uses of this technique need to be evaluated in order to verify the specificity of such a tool in identifying either functional limitation of a deficient joint or improvements during a rehabilitation program.

\section{ACKNOWLEDGMENTS}

\section{Author Contributions:}

Study concept and design: P. Picerno, G. Melchiorri.

Acquisition of data: V. Viero, T. Triossi.

Analysis and interpretation of data: P. Picerno, M. Donati.

Drafting of manuscript: P. Picerno, G. Melchiorri.

Critical revision of manuscript for important intellectual content:

V. Tancredi.

Statistical analysis: P. Picerno.

Study supervision: G. Melchiorri.

Financial Disclosures: Marco Donati, at the time of data collection, was employed by SENSORIZE S.r.l, producer of the inertial sensor used in the study. The authors have declared they were not influenced in any way by this company during the preparation of the manuscript. Funding/Support: This material was based on work supported in part by the Don Gnocchi Foundation, Milan, Italy.

Additional Contributions: The authors would like to thank the Exercise Physiology Laboratory of University of Foro Italico for its cooperation in providing and supporting experiments with the isokinetic dynamometer.

Institutional Review: This study was approved by the ethical committee of the University of Rome Tor Vergata, and informed consent was obtained from each participant.

Participant Follow-Up: The authors do not plan to inform participants of the publication of this study.

\section{REFERENCES}

1. Kulig K, Andrews JG, Hay JG. Human strength curves. Exerc Sport Sci Rev. 1984;12:417-66. [PMID:6376139] http://dx.doi.org/10.1249/00003677-198401000-00014

2. Osternig LR. Isokinetic dynamometry: Implications for muscle testing and rehabilitation. Exerc Sport Sci Rev. 1986; 14:45-80. [PMID:3525192]

3. Cadogan A, Laslett M, Hing W, McNair P, Williams M. Reliability of a new hand-held dynamometer in measuring shoulder range of motion and strength. Man Ther. 2011; 16(1):97-101. [PMID:20621547]

http://dx.doi.org/10.1016/j.math.2010.05.005

4. Durfee WK, Iaizzo PA. Rehabilitation and muscle testing. In: Webster JG, editor. Encyclopedia of medical devices and instrumentation. 2nd ed. Hoboken (NJ): John Wiley \& Sons; 2006. p. 62-71.

5. Cabri JM, Clarys JP. Isokinetic exercise in rehabilitation. Appl Ergon. 1991;22(5):295-98. [PMID:15676825] http://dx.doi.org/10.1016/0003-6870(91)90383-S 
6. Philippou A, Maridaki M, Bogdanis GC. Angle-specific impairment of elbow flexors strength after isometric exercise at long muscle length. J Sports Sci. 2003;21(10):85965. [PMID:14620029] http://dx.doi.org/10.1080/0264041031000140356

7. Roy JS, Macdermid JC, Boyd KU, Faber KJ, Drosdowech D, Athwal GS. Rotational strength, range of motion, and function in people with unaffected shoulders from various stages of life. Sports Med Arthrosc Rehabil Ther Technol. 2009;1:4. [PMID:19284527] http://dx.doi.org/10.1186/1758-2555-1-4

8. Toledo JM, Ribeiro DC, Loss JF. Mechanical criteria for progression in internal and external rotation exercises of the shoulder in the sagittal plane. Rev Bras Fisioter. 2007; 11(1):49-56. http://dx.doi.org/10.1590/S1413-35552007000100009

9. Amankwah K, Triolo RJ, Kirsch R. Effects of spinal cord injury on lower-limb passive joint moments revealed through a nonlinear viscoelastic model. J Rehabil Res Dev. 2004;41(1):15-32. [PMID:15273894] http://dx.doi.org/10.1682/JRRD.2004.01.0015

10. Nosaka K, Sakamoto K. Effect of elbow joint angle on the magnitude of muscle damage to the elbow flexors. Med Sci Sports Exerc. 2001;33(1):22-29. [PMID:11194107] http://dx.doi.org/10.1097/00005768-200101000-00005

11. Saka T, Akova B, Yazici Z, Sekir U, Gür H, Ozarda Y. Difference in the magnitude of muscle damage between elbow flexors and knee extensors eccentric exercises. J Sports Sci Med. 2009;8(1):107-15. [PMID:24150563]

12. Coombs R, Garbutt G. Developments in the use of the hamstring/quadriceps ratio for the assessment of muscle balance. J Sports Sci Med. 2002;1(3):56-62. [PMID:24701125]

13. Osternig LR, Hamill J, Lander JE, Robertson R. Co-activation of sprinter and distance runner muscles in isokinetic exercise.

Med Sci Sports Exerc. 1986;18(4):431-35.

[PMID:3747804] http://dx.doi.org/10.1249/00005768-198608000-00012

14. Bosco C. Methods of functional testing during rehabilitation exercises. In: Puddu G, Giombini A, Selvanetti A, editors. Rehabilitation of sports injuries: Current concepts. Berlin (Germany): Springer Verlag; 2001. p. 11-22.

15. Cuesta-Vargas AI, Galán-Mercant A, Williams JM. The use of inertial sensors system for human motion analysis. Phys Ther Rev. 2010;15(6):462-73. [PMID:23565045] http://dx.doi.org/10.1179/1743288X11Y.0000000006

16. Luinge HJ, Veltink PH. Measuring orientation of human body segments using miniature gyroscopes and accelerometers. Med Biol Eng Comput. 2005;43(2):273-82.

[PMID:15865139]

http://dx.doi.org/10.1007/BF02345966
17. Tranquilli C, Bernetti A, Picerno P. Ambulatory joint mobility and muscle strength assessment during rehabilitation using a single wearable inertial sensor. Med Sport (Roma). 2013;66(4):583-97.

18. Parel I, Cutti AG, Fiumana G, Porcellini G, Verni G, Accardo AP. Ambulatory measurement of the scapulohumeral rhythm: Intra- and inter-operator agreement of a protocol based on inertial and magnetic sensors. Gait Posture. 2012;35(4):636-40. [PMID:22300730]

http://dx.doi.org/10.1016/j.gaitpost.2011.12.015

19. El-Gohary M, McNames J. Shoulder and elbow joint angle tracking with inertial sensors. IEEE Trans Biomed Eng. 2012;59(9):2635-41. [PMID:22911538] http://dx.doi.org/10.1109/TBME.2012.2208750

20. Squadrone R, Rodano R, Preatoni E. Comparison of velocity and power output data derived from an inertial based system and an optical encoder during squat lifts in a weight room setting. J Sports Med Phys Fitness. 2012;52(1):40-46. [PMID:22327085]

21. Wakeling JM, Nigg BM. Soft-tissue vibrations in the quadriceps measured with skin mounted transducers. J Biomech. 2001;34(4):539-43. [PMID:11266679] http://dx.doi.org/10.1016/S0021-9290(00)00203-7

22. Forner-Cordero A, Mateu-Arce M, Forner-Cordero I, Alcántara E, Moreno JC, Pons JL. Study of the motion artefacts of skin-mounted inertial sensors under different attachment conditions. Physiol Meas. 2008;29(4):N21-31. [PMID:18401071] http://dx.doi.org/10.1088/0967-3334/29/4/N01

23. Favre J, Aissaoui R, Jolles BM, Siegrist O, de Guise JA, Aminian K. 3D joint rotation measurement using MEMs inertial sensors: Application to the knee joint. In Proceedings of Ninth International Symposium on the 3D Analysis of Human Movement. International Society of Biomechanics (ISB) Technical Group on the 3-D Analysis of Human Movement; 2006; Valenciennes, France.

24. Woodman OJ. An introduction to inertial navigation. Technical Report UCAM-CLTR-696. Cambridge (UK): University of Cambridge Computer Laboratory; 2007.

25. Mahony R, Hamel T, Pflimlin JM. Nonlinear complementary filters on the special orthogonal group. IEEE Trans Automat Contr. 2008;53(5):1203-18. http://dx.doi.org/10.1109/TAC.2008.923738

26. Woltring HJ. On optimal smoothing and derivative estimation from noisy displacement data in biomechanics. Hum Mov Sci. 1985;4:229-45. http://dx.doi.org/10.1016/0167-9457(85)90004-1

27. de Leva P. Adjustments to Zatsiorsky-Seluyanov's segment inertia parameters. J Biomech. 1996;29(9):1223-30.

[PMID:8872282]

http://dx.doi.org/10.1016/0021-9290(95)00178-6 
28. Shrout PE, Fleiss JL. Intraclass correlations: Uses in assessing rater reliability. Psychol Bull. 1979;86(2):420-28. [PMID:18839484] http://dx.doi.org/10.1037/0033-2909.86.2.420

29. Evans K, Horan SA, Neal RJ, Barrett RS, Mills PM. Repeatability of three-dimensional thorax and pelvis kinematics in the golf swing measured using a field-based motion capture system. Sports Biomech. 2012;11(2):262-72. [PMID:22900406] http://dx.doi.org/10.1080/14763141.2012.654502

30. Besier TF, Sturnieks DL, Alderson JA, Lloyd DG. Repeatability of gait data using a functional hip joint centre and a mean helical knee axis. J Biomech. 2003;36(8):1159-68. [PMID:12831742] http://dx.doi.org/10.1016/S0021-9290(03)00087-3

31. Kavanagh JJ, Morrison S, James DA, Barrett R. Reliability of segmental accelerations measured using a new wireless gait analysis system. J Biomech. 2006;39(15):2863-72. [PMID:16253263] http://dx.doi.org/10.1016/j.jbiomech.2005.09.012

32. Kadaba MP, Ramakrishnan HK, Wootten ME, Gainey J, Gorton G, Cochran GV. Repeatability of kinematic, kinetic, and electromyographic data in normal adult gait. J Orthop Res. 1989;7(6):849-60. [PMID:2795325] http://dx.doi.org/10.1002/jor.1100070611

33. O’Dwyer N, Smith R, Halaki M, Rattanaprasert U. Independent assessment of pattern and offset variability of time series waveforms. Gait Posture. 2009;29(2):285-89.
[PMID:18976919]

http://dx.doi.org/10.1016/j.gaitpost.2008.09.005

34. Luo J, Bai J, Shao J. Application of the wavelet transforms on axial strain calculation in ultrasound elastography. Prog Nat Sci. 2006;16(9):942-47. http://dx.doi.org/10.1080/10020070612330093

35. Kuhlman JR, Iannotti JP, Kelly MJ, Riegler FX, Gevaert ML, Ergin TM. Isokinetic and isometric measurement of strength of external rotation and abduction of the shoulder. J Bone Joint Surg Am. 1992;74(9):1320-33.

[PMID:1429787]

Submitted for publication June 17, 2014. Accepted in revised form January 26, 2015.

This article and any supplementary material should be cited as follows:

Picerno P, Viero V, Donati M, Triossi T, Tancredi V, Melchiorri G. Ambulatory assessment of shoulder abduction strength curve using a single wearable inertial sensor. J Rehabil Res Dev. 2015;52(2):171-80.

http://dx.doi.org/10.1682/JRRD.2014.06.0146

ResearcherID: Pietro Picerno, PhD: D-7603-2015.

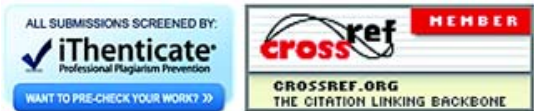


